\title{
On-chip Stimuli Generation for ADC Dynamic Test by $\Sigma \Delta$ Technique
}

\author{
Shakeel Ahmad and Jerzy Dąbrowski \\ Department of Electrical Engineering \\ Linköping University \\ 58183 Linköping, Sweden \\ e-mail: \{shakeel, jdab\}@isy.liu.se
}

\begin{abstract}
This paper presents application of the $\Sigma \Delta$ modulation technique to the on-chip dynamic test for $A / D$ converters. The wanted stimulus such as a single- or two-tone signal is encoded into one-bit $\Sigma \Delta$ sequence, which after simple low-pass filtering is applied to the circuit under test with low noise and without distortion. In this way a large dynamic range is achieved making the performance harmonic- and intermodulation dynamic test viable. By a systematic approach we select the order and type of a $\Sigma \Delta$ modulator, and develop the frequency plan suitable for spectral measurements on a chip. The technique is illustrated by simulation of a practical ADC under test.
\end{abstract}

Keywords-Stimuli generation; on-chip test; $\Sigma \Delta$ modulation

\section{INTRODUCTION}

With increased complexity of the contemporary ICs the mixed-signal test is becoming more cumbersome and costly. Production test techniques using test access points are often limited by chip performance, area overhead, and cost of the test instrumentation. For this reason design-for-testability (DfT) and built-in-self-test (BiST) techniques for mixed-signal ICs have been around for the last decade [1]. With the advent of embedded processors the mixed-signal/RF BiST on a chip has become viable. However, the practical implementations are still a challenge [2].

In this paper we address the on-chip stimuli generation, essential for BiST. The spectral purity and high SNR of the generated signals are the main objectives. Spectrally pure stimuli can be derived from 1-bit sequences using modulation techniques like PWM [3] or $\Sigma \Delta$ [4] implemented in software. In this case the D/A conversion can be achieved by simple low-pass filtering thus avoiding nonlinear distortions. In PWM approach the inherent high frequency components related to the PWM carrier frequency $f_{c}$, can be suppressed effectively with a passive analog filter. However, the associated quantization noise undergoes folding and the resulting FFT noise floor is proportional to $f_{c} /\left(N f_{c l k}\right)$, where $N$ is the FFT length and $f_{c l k}$ stands for the clock (sampling) frequency of the system. A satisfactory noise floor level can be achieved at the expense of a large value of the product $N f_{c l k}$ since the carrier $f_{c}$ must be (at least) by one order of magnitude larger than the frequency of the encoded signal, $f_{0}$. Pushing $f_{c l k}$ to practical limits, say in a $\mathrm{GHz}$ range, might not be sufficient resulting in long FFT sequences that claim more test time.

To alleviate the problem the noise shaping technique based on $\Sigma \Delta$ modulation can be used as proposed in [4]. The quantization noise floor is largely reduced in this way providing a large dynamic range for spectral measurements. Here, we investigate different variants of the $\Sigma \Delta$ modulation in terms of the dynamic test especially for high performance $A / D$ converters. For this purpose we specify the frequency measurement bands, and by a systematic approach choose the stimuli frequencies and select the order and type of a $\Sigma \Delta$ modulator suitable for spectral measurements. The harmonicand intermodulation distortion measurements ( $H D \#$ and $I M \#)$ are the primary concern in this case. By careful frequency planning a possible masking of spurious tones in the test response is avoided. We show that with a reasonable overhead, i.e. using moderate values of $N$ and $f_{c l k}$, high dynamic range measurements are viable. The method is validated by simulation of a practical ADC under test where the limitations due the ADC quantization effects are revealed. Especially the frequency selective measurements using band-pass $\Sigma \Delta$-encoded stimuli are limited in this way.

\section{StimUli ENCODING AND DyNAMIC RANGE}

Consider a 1-bit signal from a low-pass $\Sigma \Delta$ modulator. If it is software generated its whole noise is the quantization noise. For the generic model of $L$-th order modulator the discrete noise transfer function is given by [5]:

$$
\operatorname{NTF}(z)=\left(1-z^{-1}\right) L
$$

Consequently, the power spectral density of the quantization noise can be estimated from:

$$
S_{q}(f)=\left|N T F\left(e^{j 2 \pi f / f_{S}}\right)\right|^{2} S_{n}(f)
$$

where $S_{n}(f)=\Delta^{2} /\left(12 f_{S}\right)$ is assumed constant over the Nyquist band, $\Delta$ is the quantizer resolution and $f_{S}$ is the sampling (or clock) frequency. When using FFT with spectral resolution $\Delta f$ the resulting noise floor can be expressed as: 


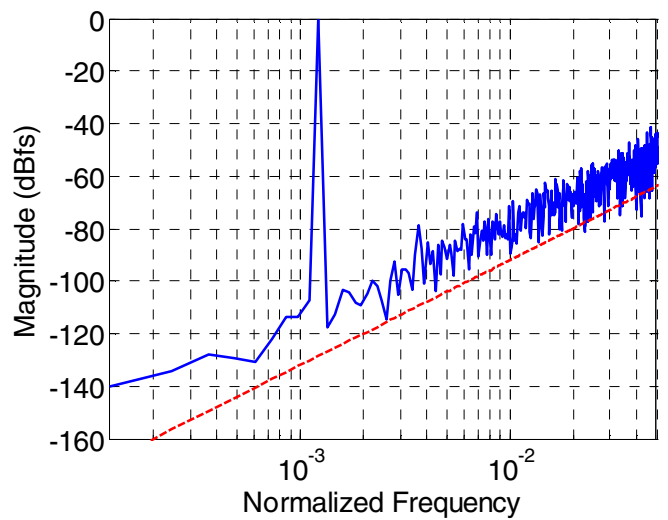

Fig. 1. $\Sigma \Delta$ encoded tone spectrum $(L=2)$

$$
P_{q}(f)=\int_{f-\Delta f / 2}^{f+\Delta f / 2} S_{q}(f) d f
$$

For large oversampling ratios the noise floor (3) can be calculated as:

$$
P_{q}(f) \cong \frac{\Delta^{2}}{12 N}\left(\frac{2 \pi f}{f_{S}}\right)^{2 L}
$$

where $N$ is the FFT length and $\Delta f=f_{S} / N$ is the spectral resolution. As seen, the noise floor is mostly affected by the oversampling ratio and the modulator order.

For the purpose of spectral measurements we can define the dynamic range $D R(f)$ using $-3 \mathrm{~dB}$ FS $\Sigma \Delta$-encoded tone with amplitude $\Delta /(2 \sqrt{ } 2)$ (to avoid overloading):

$$
D R(f)=\frac{3 N}{4}\left(\frac{f_{S}}{2 \pi f}\right)^{2 L}
$$

which in a more practical $\mathrm{dB}$ scale is:

$$
D R(f) \cong 20 L \log \frac{f_{S}}{f}-16 L+10 \log N-1.2 \mathrm{~dB}
$$

For comparison a spectrum of $\Sigma \Delta$-encoded tone ( $-3 \mathrm{~dB} F S)$ is shown in Fig. $1(L=2$ and $N=8192)$. To avoid spectral leakage the signal frequency is chosen to fit one FFT bin. The discrepancy between the spectrum expected from (4-5) and the simulated spectrum can be attributed to the linear scaling effect of the quantizer gain and the randomness of the quantization noise [5, Ch. 4.2]. To reduce the noise leakage effect and achieve a better match between the simulated- and the theoretical spectrum, windowing can be used. In Fig. 2 the FFT spectrum of the same signal with Hann window is shown. In this case the spectral resolution is $\Delta f=3 f_{S} /(2 N)$ so the estimate (5) is corrected accordingly (by subtracting $1.76 \mathrm{~dB}$ ).

The above results show that stimuli encoded by 1-bit lowpass $\Sigma \Delta$ are well suited for spectral test at lower frequencies, where a high dynamic range is achieved. When the modulator order is increased by 1 the $D R(f)$ is expected to improve by $20 \log \left(f_{S} / f\right)-16 \mathrm{~dB}$, but in practice it is much less because of the quantization noise leakage in FFT.

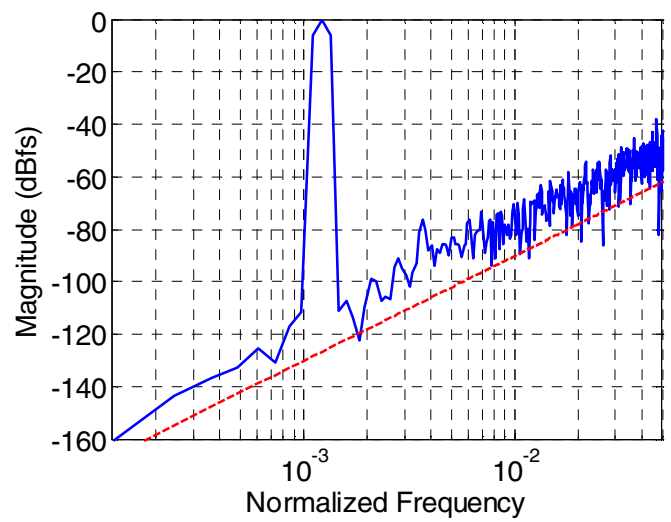

Fig. 2. $\Sigma \Delta$ encoded tone windowed spectrum $(L=2)$

Before a 1-bit stimulus is applied to the circuit the high frequency spectral components must be effectively suppressed with a low pass linear filter (reconstruction filter) with cut-off frequency above the encoded signal. In practice, it can be a simple passive RC filter integrated on a chip. When harmonic distortions are to be measured using this stimulus, they will experience increasingly higher noise floor. Specifically, by using $L$-th order encoded signal and a 2 nd order low-pass filter the noise floor would rise by $20(L-2) \mathrm{dB} / \mathrm{dec}$ of frequency. So the 2nd harmonic would suffer from $6(L-2) \mathrm{dB}$ higher noise floor than the fundamental, and the 3rd harmonic $9.5(L-2) \mathrm{dB}$, respectively. Since for performance measurements $L=3, \ldots 5$ would be preferred while the filter order might be still 2 , this increase of the noise floor can largely reduce the dynamic range and even obscure the harmonic distortion measurement. Obviously the frequency range below the fundamental appears more attractive. To make use of it the harmonic test can be replaced by the 2-tone intermodulation test.

A two-tone stimulus encoded by 2 nd order modulator is considered as an example. To avoid overloading of the modulator the tones are set $P_{\text {in }}=-12 \mathrm{~dB}$ FS each and the stimulus after going through a low-pass filter (2nd order) is applied to a generic weakly nonlinear block defined as $x_{\text {out }}=x_{\text {in }}-a x_{\text {in }}^{3}$ where $a=10^{-3}$. In the spectral response shown in Fig. 3 one of the intermodulation tones is well seen some $20 \mathrm{~dB}$ above the noise floor while the other nonlinear components are obscured. The 3rd order intermodulation distortion is evident, $I M 3 \cong-87 \mathrm{~dB}$ and it is close to the predicted value $I M 3=20 \log (3 a / 4)+2 P_{\text {in }} \cong-86.5 \mathrm{~dB}$.

Observe that when the measurement frequency $f$ is moved by one octave up the noise floor will be higher by $6 L=18 \mathrm{~dB}$ and the IM3 tone will be obscured. The frequency limit imposed on the measurement band including $6 \mathrm{~dB}$ reserve can be derived from the condition:

$$
P_{q}(f)<P_{i n}+I M 3(f)-6 \mathrm{~dB}
$$

which by using (5) can be rewritten as:

$$
\log \frac{f}{f_{S}}<\frac{I M 3(f)+10 \log N-16.2}{20 L}-0.8 \mathrm{~dB}
$$

where we assumed $P_{\text {in }}=-12 \mathrm{~dB}$ FS. Having specified the IM3 


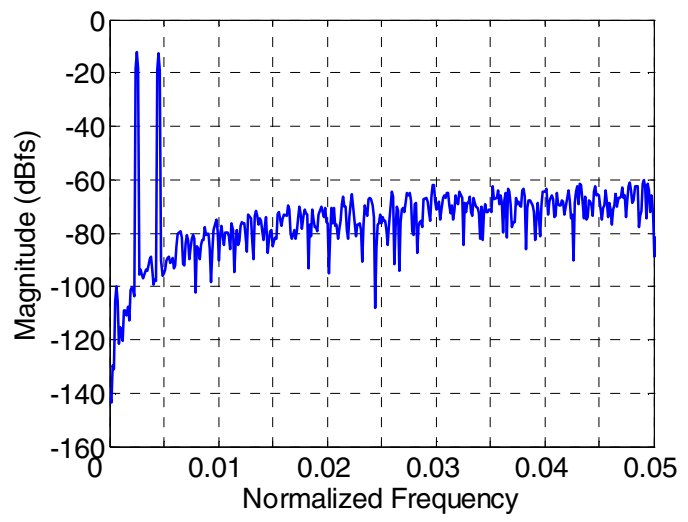

Fig. 3. Two-tone test response using LP $\Sigma \Delta$ with $L=2$

measurement range the frequency upper bound $f_{m x} / f_{S}$ can be estimated with respect to the modulator order and FFT length. In a design perspective when we also specify the frequency upper bound the modulator order $L$ can be estimated vs. $N$ as shown in Fig. 4 where we assumed the $I M 3$ range $100 \mathrm{~dB}$. For example, for $f_{m x} / f_{S}=0.01$ and $L=3$ the required $N \geq 2^{15}$ but for $f_{m x} / f_{S}=0.005$ and $L=3$ only $N=2^{9}$ is sufficient.

\section{FREQUENCY SELECTIVE MEASUREMENTS}

By using band-pass $\Sigma \Delta$ modulation the quantization noise stop-band can be placed at any non-zero frequency $f_{0}<f_{\mathrm{S}} / 2$ that makes spectral measurements at higher frequencies viable. A high dynamic range $D R\left(f_{0}\right)$ is achieved by notch-shaped transfer function with zero-gain at $f_{0}$. In fact, $D R\left(f_{0}\right)$ is limited by the FFT noise leakage which largely elevates the noise floor in the stop-band (Fig. 5). There is no simple analytical model to quantify this effect. In practice the $D R\left(f_{0}\right)$ of the 4th order BP $\Sigma \Delta$ is comparable with $D R(f)$ of the 2 nd order LP $\Sigma \Delta$ for frequencies close to zero. By increasing the modulator order from 2 to 4 the noise floor at $f_{0}$ drops by almost $60 \mathrm{~dB}$.

The BP $\Sigma \Delta$ encoding can be used both for the harmonic and intermodulation distortion test with the measurement band at $f_{0}$. The frequency test plan depends on the reconstruction filter used to suppress spectral replicas of the stimulus. To achieve $\geq 40 \mathrm{~dB}$ attenuation with 2 nd order filter, a span of at least one decade between the test tone and its first FFT replica is

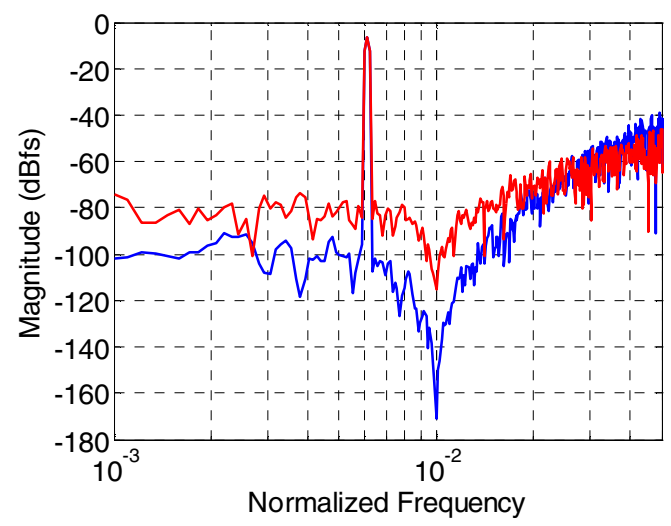

Fig. 5. Tone stimulus encoded by 2 nd and 4 th order BP $\Sigma \Delta$

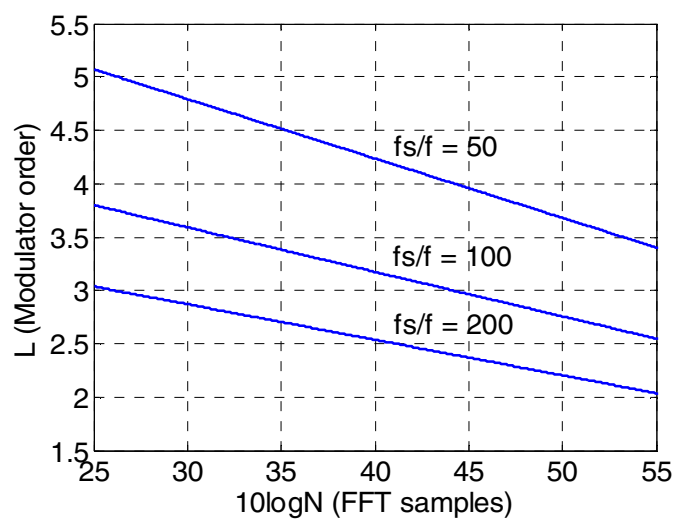

Fig. 4. Modulator order vs. FFT length for different measurement bands

required. To measure the $k$ th harmonic the tone stimulus should be placed at $f_{0} / k$ so we have:

$$
\left(f_{S}-f_{0} / k\right)-f_{0} / k>10 f_{0} / k
$$

which means $f_{0} / f_{S}<k / 12$ where $f_{0} / k$ should fall in one FFT bin. For example, for 2 nd order harmonic distortion HD2 test we can choose $f_{0} / f_{S}=2 / 16$ and $f_{1} / f_{S}=2 / 32$, accordingly. This frequency plan is used to measure $H D 2$ response of a generic nonlinear block defined as $x_{\text {out }}=x_{\text {in }}-a x_{\text {in }}^{2}$ with $a=10^{-3}$ as shown in Fig. 6. The simulated HD2 $\cong-73 \mathrm{~dB}$ while the predicted value would be $H D 2=20 \log a-6+P_{\text {in }} \cong-72 \mathrm{~dB}$.

For the intermodulation test a similar frequency plan can be derived. For two tones at $f_{1}$ and $f_{2}$ (where $f_{1}<f_{2}<f_{0}$ ) we find $f_{2} / f_{S}<1 / 12$ and $f_{0}=2 f_{2}-f_{1}$.

\section{APPLICATION EXAMPLE}

We consider a 10-bit time-interleaved ADC with $1 \mathrm{GHz}$ sampling frequency in a test setup shown in Fig. 7 [4]. The test at low frequencies will be completed by the two-tone stimulus encoded by LP $\Sigma \Delta$. As the expected $S N R_{m x}$ of this ADC would be $6 \times 10+1.76 \mathrm{~dB}$ then the corresponding DR would be $S N R_{m x}$ $+10 \log \left(f_{S} /(2 \Delta f)\right)$ where $\Delta f$ is the FFT spectral resolution. For $N$ samples with the Hann window we achieve $D R^{*} \cong 61.8+$ $10 \log (N / 3)$. Using two-tone stimulus with $P_{\text {in }}=-12 \mathrm{~dB}$ FS the $I M 3(f)$ range can be taken as $-\left(D R^{*}+P_{\text {in }}\right) \cong-10 \log N-45 \mathrm{~dB}$. When substituted to (7) including $10 \mathrm{~dB}$ reserve for the noise

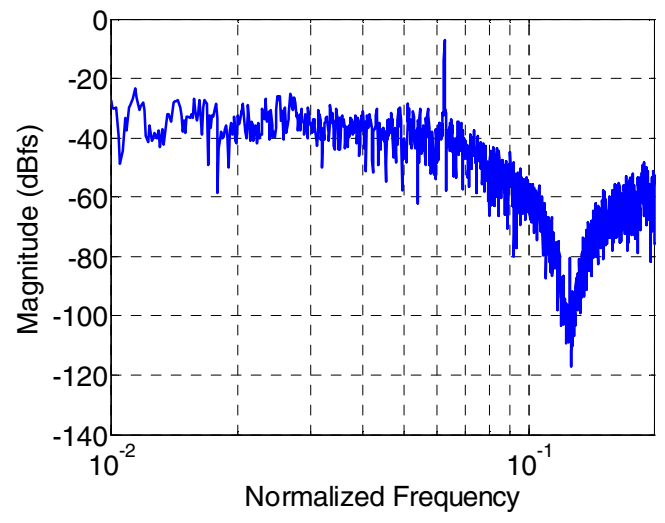

Fig. 6. $H D 2$ test with BP $\Sigma \Delta$-encoded stimulus 


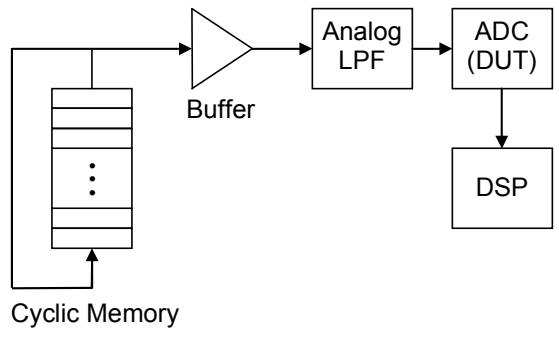

Fig. 7. Test setup with stimulus stored in cyclic memory.

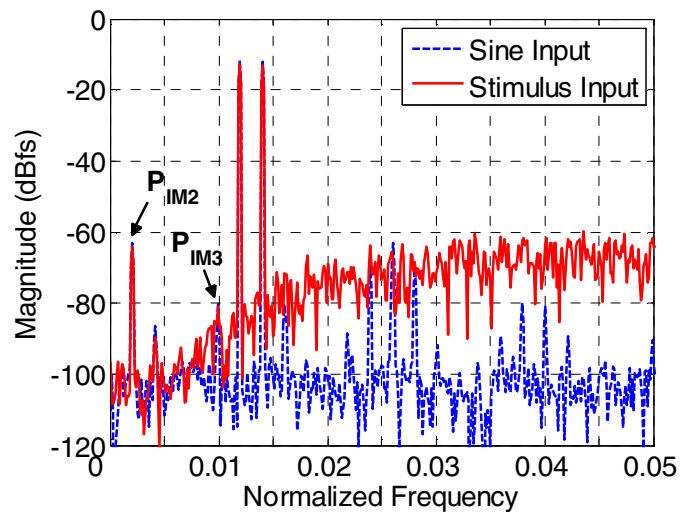

Fig. 8. ADC two-tone response for IM2/IM3 test.

leakage effects it gives:

$$
\log \left(f / f_{S}\right)<-71.2 / 20 L-0.8 \mathrm{~dB}
$$

Choosing $L=3$ or $L=4$ we find the measurement band $f<0.01 f_{S}$ or $f<0.02 f_{S}$, respectively. Moreover, in the frequency plan we have to avoid a potential interference between IM3 and IM2 tones which appear at $\left(2 f_{1}-f_{2}\right)$ and $\left(f_{2}-f_{1}\right)$, respectively. Hence, we infer that $f_{1} / f_{2} \neq 2 / 3$ should be satisfied.

In Fig. 8 the $I M 2 / I M 3$ test of the ADC is shown for $L=3$ where $f_{I M 2}=2 \mathrm{MHz}$ and $f_{I M 3}=10 \mathrm{MHz}$. A 2nd order LP filter with $f_{T}=30 \mathrm{MHz}$ is used. For comparison a response to the noiseless two-tone signal is also shown. The achieved accuracy for $I M 2$ and $I M 3$ is better than $1 \mathrm{~dB}$.

Going towards higher frequencies as required for $H D \#$ test we use a 4th order BP $\Sigma \Delta$-encoded tone. For the tone at $50 \mathrm{MHz}$ the notch at $f_{0}=100 \mathrm{MHz}$ is used to measure $H D 2$ as shown in Fig. 9. The cutoff frequency of the reconstruction filter is $80 \ldots 120 \mathrm{MHz}$. The measurement accuracy of $H D 2$ is as in the IM2/IM3 test.

In a similar way a notch placed at $f_{0}=150 \mathrm{MHz}$ should enable the HD3 measurement. In this case however, the stimulus noise power is increased at low frequencies, degrading SNR at the ADC input. The ADC tends to spread the noise uniformly in frequency and the notch tends to vanish as shown in Fig. 10. Using a more efficient LP filter or a higher order BP $\Sigma \Delta$ which offers a deeper notch at $f_{0}$ does not help in this respect. This is unlike the model demonstrated in Fig. 6 where the notch is not affected. We observed that an $\mathrm{ADC}$ under test can preserve the notch for $f_{0}>0.1 f_{S}$ when SNR is improved by noise shaping introduced also at low frequencies.

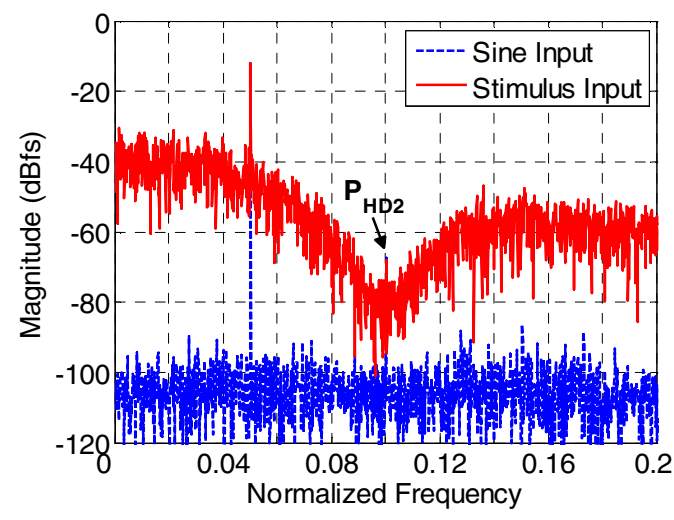

Fig. 9. ADC harmonic response for HD2 test

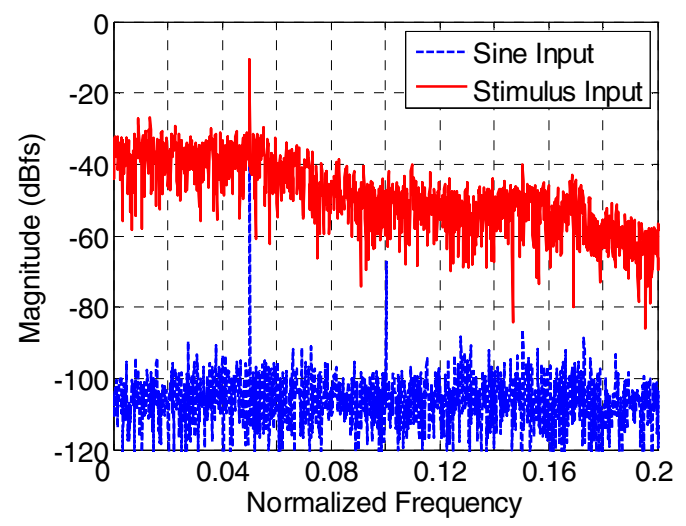

Fig. 10. ADC harmonic response for HD3 test

\section{CONCLUSION}

By using 1-bit $\Sigma \Delta$ modulation, spectrally pure stimuli with a high dynamic range can be generated. In this way ADC dynamic test such as $H D \#$ or $I M \#$ can be carried out in a simple setup. The FFT artifacts can be avoided by careful frequency planning both for low- and band-pass $\Sigma \Delta$ encoding technique. The latter enables spectral measurements also at higher frequencies taking advantage of notch-shaped stimuli spectra. However, when the notch frequency goes up the unfiltered portion of the quantization noise tends to decrease SNR which ultimately appears a hindrance for the ADC test in this case. A noise shaping which is more sophisticated than offered by standard LP or BP $\Sigma \Delta$ technique is required in this case that we consider as a direction for the future work.

\section{REFERENCES}

[1] M. L. Bushnell, V. D. Agrawal, Essentials of Electronic Testing, Kluwer Academic Publishers, 2004

[2] J. L. Huertas (Ed.), Test and Design-for-Testability in Mixed-Signal Integrated Circuits, Springer, 2004.

[3] S. Ahmad, J. Dąbrowski, "ADC on-Chip Dynamic Test by PWM Techniques," Int. Conf. on Signals and Electronic Systems, 2008.

[4] M. M. Hafed et al. "A 4-GHZ Effective Sample Rate Integrated Test Core for Analog and Mixed Signal Circuits," IEEE J. Solid-State Circuits, vol. 37, no. 4, April 2002, pp.499-514.

[5] R. Schreier, G. C. Temes, Understanding Delta-Sigma Data Converters, John Wiley \& Sons, 2005. 